\title{
High Spatial Resolution Spectroscopy of W51 IRS2E and IRS2W: Two Very Massive Young Stars in Early Formation Stages
}

\author{
Cássio L. Barbosa ${ }^{1}$ \\ IPEDD - UNIVAP, Av. Shishima Hifumi, 2911, 12244-000 São José dos Campos, SP, Brazil \\ cassio@univap.br \\ Robert D. Blum² \\ NOAO Gemini Science Center, 950 Cherry Ave., Tucson, AZ 85719, USA \\ rblum@noao.edu \\ Peter S. Conti ${ }^{3}$ \\ JILA, University of Colorado, Boulder, CO 80309-0440, USA \\ pconti@jila.colorado.edu \\ Augusto Damineli ${ }^{4}$ \\ IAG - USP. Rua do Matão, 1226, 05508-900, São Paulo, SP, Brazil \\ damineli@astro.iag.usp.br \\ and \\ Elysandra Figuerêdo ${ }^{5}$ \\ The Open University - Walton Hall, Milton Keynes MKr7 6AA, UK \\ e.figueredo@open.ac.uk
}

\begin{abstract}
We present $K$-band spectra of the near infrared counterparts to IRS2E and IRS2W which is associated with the ultracompact HII region W51d, both of them embedded sources in the Galactic compact HII region W51 IRS2. The high spatial resolution observations were obtained with the laser guide star facility and Near infrared Integral Field Spectrograph (NIFS) mounted at the Gemini North observatory. The spectrum of the ionizing source of W51d shows the photospheric
\end{abstract}


features NIII (21155 $\AA$ ) in emission and HeII (21897 $\AA$ ) in absorption which lead us to classify it as an young O3 type star. We detected CO overtone in emission at $23000 \AA$ in the spectrum of IRS2E, suggesting that it is a massive young object still surrounded by an accretion disc, probably transitioning from the hot core phase to an ultracompact HII region.

Subject headings: stars: early type — stars: formation — infrared: stars — techniques: spectroscopy

\section{Introduction}

The mechanisms of massive star formation $\left(M_{*}>10 M_{\odot}\right)$ are the subject of intense discussion even after two decades of great progress and improvement of observational techniques. In the last two decades, the development of larger and more sensitive infrared detectors associated with the commissioning of 8-m class telescopes equipped with adaptive optics has made it possible to observe ever deeper into the sites of massive star formation at relatively short wavelengths. Yet, while all these improvements have contributed to our understanding of massive star formation, the basic process, i.e., formation via an accretion disk, coalescence, competitive accretion, or a combination of these is still unknown (for a review on this discussion see Zinnecker \& Yorke 2007). The Spitzer Space Telescope is providing an unprecedented view at longer wavelengths and soon the Herschel Space Observatory and ALMA will combine very high sensitivity and angular resolution to probe the earliest phases of massive star formation.

W51 is one of the most luminous complexes of massive star forming regions in the Galaxy (Goldader \& Wynn-Williams 1994) with multiple HII regions (Wilson et al. 1970) that host at least six HII regions with embedded clusters, all of them optically obscured (Kumar et al. 2004). The most luminous of all HII regions is G49.5-0.4 which can be resolved into mainly two bright infrared sources: IRS1 and IRS2, which are coincident with radio sources W51e and W51d respectively (Martin 1972). IRS2 is a compact HII region harboring three ultracompact HII (UCHII) regions W51d, W51d $1_{1}$ and $W 51 \mathrm{~d}_{2}$ (Mehringer 1994) and a plethora of infrared sources (Goldader \& Wynn-Williams 1994; Okumura et al. 2001; Okamoto et al. 2001; Kramer et al. 2001; Figuerêdo et al. 2008; Barbosa et al. 2008). The most prominent sources in the $K$ band are named IRS2 East and IRS2 West (or IRS2E and IRS2W, respectively, for short). IRS2W was identified as a NIR and MIR peak of nebular emission (Goldader \& Wynn-Williams 1994) and Okamoto et al. (2001) have correlated it with the UCHII region W51d. 
In this letter we report the results of spectroscopic observations taken with laser guide star (LGS) adaptive optics of IRS2E and the UCHII region W51d (IRS2W). In the section 2 we report the technical details of the observations and in the section 3 we report and discuss the results. Finally, in the section 4 we summarize our conclusions.

\section{Observations}

The data were obtained with the Near infrared Integral Field Spectrograph (NIFS) mounted on the Frederick C. Gillett 8-m telescope at the Gemini North observatory on Mauna Kea, HI, USA, in queue mode on May, 4th 2007 under program ID GN-2007A-Q-34. NIFS provides 2D integral field spectroscopy with $\mathrm{R} \sim 5200$ over a $3^{\prime \prime} \times 3^{\prime \prime}$ field of view with $0.043 \times 0.10$ " rectangular "pixels". Also, we used the Gemini North Adaptive Optics system Altair with a LGS to achieve an angular resolution of $<0.2^{\prime \prime}$ measured upon the $2 \mathrm{D}$ images build from the datacubes.

The observations were carried out with the standard procedures for the near infrared (NIR): we observed IRS2 in a series of three pointings of 1000 seconds on source and 1000 seconds on a "blank" sky position at $\sim 10$ " north of IRS2 for each field. The latter image was used to subtract the sky emission from the images of the targets. We also observed the A0V star HIP 98640 with similar airmass as ISRS2 in order to remove the telluric absorption lines from the spectra of the observed targets. The spectra of A0V stars show only the $\operatorname{Br} \gamma$ feature in absorption in the $K$ band. We eliminated this feature by fitting a Voight profile to the $\mathrm{Br} \gamma$ leaving a spectrum with only telluric absorption features. This telluric spectrum was used to correct the spectra of the targets.

Wavelength calibration was achieved with an exposure of an Argon arc lamp obtained with the Gemini calibration module GCAL. All data were precessed under IRAF1 environment with scripts written for NIFS available through the Gemini IRAF package. We extracted the spectra through a $0.4^{\prime \prime}$ circular aperture centered on the sources. This aperture was chosen to minimize the contribution of the nebular emission from the HII region.

We show in Figure 1 the finding chart of IRS2. It is composed by the high resolution image taken with the adaptive optics NIR camera NACO in the $K$ band. This image is available at the ESO public database. We overplotted the radio continuum emission at 2

\footnotetext{
${ }^{1}$ IRAF is distributed by the National Optical Astronomy Observatories, which are operated by the Association of Universities for Research in Astronomy, Inc., under cooperative agreement with the National Science Foundation.
} 
cm from Wood \& Churchwell (1989) in white contours. The black contours represent the MIR emission at $12 \mu \mathrm{m}$ from the Gemini South MIR camera T-ReCS. All NIFS pointings are represented by white squares and the sources which we have spectra are labeled. In the following section we present the results of the observations of IRS2E and the UCHII region W51d in the "pseudo long-slit" mode extracted from the images delivered by NIFS. A complete analysis of these data, including the spectra of all observed sources and the results from the IFU mode, such as the velocity maps of the regions observed will be presented in a forthcoming paper (Barbosa et al. 2008).

\section{Results and Discussion}

\subsection{IRS2E}

Figure 2 shows the spectrum of IRS2E, the brightest $K$-band source in the compact HII region. It was obtained from the first pointing of the telescope. The continuum shows a steep, rising slope to longer wavelengths reflecting the fact that this source is under a heavy extinction: $\mathrm{A}_{V} \sim 63 \mathrm{mag}$ according to our unpublished MIR data. This high extinction makes IRS2E undetected at wavelengths shorter than $16000 \AA 2$. The spectrum of Figure 2 shows strong lines of HeI (20587 $\AA$ ) and unresolved $\operatorname{Br} \gamma(21661 \AA)$ that may arise from the circumstellar gas heated by hot stars. We also note nebular lines of HeI $(21127 \AA)$ and the $\mathrm{H}_{2}$ (1-0 (S1), $21128 \AA$ ) that come from the diffuse nebular emission of the HII region. Previous authors (e.g. Okumura et al. 2001; Figuerêdo et al. 2008) describe the presence of [FeIII] lines in the spectrum of IRS2E. These emission lines are typical of shocked gas in the vicinity of MYSOs (Hanson et al. 2002; Bik et al. 2006). The spectrum in the Figure 2 does not show any of these lines. This is due to the small aperture used to extract the spectrum. Actually, our unpublished line maps show that [FeIII] emission comes from the extended region between IRS2E and OKYM2/KJD5.

More interesting, IRS2E shows CO overtone emission as we detected the (2-0), (3-1), (4-2) and (5-3) bandheads between $22935 \AA$ and $23838 \AA$. Previous NIR spectroscopy of IRS2E (e.g., Okumura et al. 2001; Figuerêdo et al. 2008) could not detect the CO bandhead emission due to the large contribution of nebular emission through wide and long slits.

The detection of the $\mathrm{CO}$ overtone in emission is associated with the presence of an accretion disc, both in low-mass (e.g., Najita et al. 1996) and high-mass YSOs (Bik \& Thi

\footnotetext{
${ }^{2}$ Unpublished images obtained from the AO assisted camera NACO at the VLT shows that the source detected at the $J$ band by previous authors is just a bright knot of gas.
} 
2004; Blum et al. 2004). The CO overtone emission comes from the inner parts a circumstellar disk, in regions of high column densities $\left(10^{20}-10^{21} \mathrm{~cm}^{2}\right)$ and temperatures between $1500-4500 \mathrm{~K}$.

IRS2E is a deeply embedded source which is bright at wavelengths longer than $20000 \AA$ and is not associated with any UCHII region. These characteristics are those expected for a very young and massive YSO that is transitioning to an UCHII region; the high accretion rate presumably prevents the formation of an UCHII region as the ionized gas near the source falls onto the star, according to the evolutionary scenario proposed by Churchwell (2000). This class of object was observed in NGC 3576 by Barbosa et al. (2003), for example.

\subsection{W51d}

Figure 3 shows the spectrum of the UCHII region W51d. Treated as a peak of nebulosity in the NIR by Goldader \& Wynn-Williams (1994), Figuerêdo et al. (2008) identified a stellar object as responsible for the ionization of this region based on the correlation between the radio and MIR emission presented by Okamoto et al. (2001) and the new high resolution images obtained by us or collected at public databases.

The spectrum of W51d was normalized and zoomed near the intense Br $\gamma$ line to better show the detected features. This line has a broad component $\left(\sim 700 \mathrm{~km} \mathrm{~s}^{-1}\right)$, probably originated in fast winds coming from the hot ionizing star and two scenarios are possible to explain it. In the first case, the very young star has strong winds that blew away its surrounding dust. In the second case, the ionizing star is still surrounded by a torus that is seen more face on. However, only higher resolution data could favor one of these scenarios. Besides this nebular line, we detected photospheric lines corresponding to the NIII multiplet near $21155 \AA$ in emission and HeII at $21897 \AA$ in absorption.

The ionizing source of W51d can be classified as an early O star, given the detected features in the spectrum of Figure 3. Comparing this spectrum with the catalog of spectra of optically visible OB stars presented by Hanson et al. (2005) we can constrain its spectrum type to an O3-4 type star. The spectrum of an optically visible O3V star shows both the NIII multiplet (in emission) and the HeII (in absorption) at wavelengths longer than 20000 A. Although our spectrum cannot establish its spectral subtype, the absence of CIV (20780 $\AA$ ) in emission, as seen in mid type $\mathrm{O}$ stars, favors the identification of this object as a star hotter and more massive than type $\sim \mathrm{O} 4$. It would make this object one of the most massive ionizing source of an UCHII region (Watson \& Hanson 1997; Hanson et al. 2002; Bik et al. 2005; Blum \& McGregor 2008). 
Wood \& Churchwell (1989) and Mehringer (1994) classify the ionizing source of W51d as an $\mathrm{O} 5.5$ and $\mathrm{O} 5$ respectively, both based on radio continuum emission assuming a distance of $7 \mathrm{kpc}$ to this source. The disagreement between the results based on radio data and the spectroscopy can be explained if the massive YSO had enough time to clear the circumstellar material, lowering its density (and the extinction as well). In this case, the star will not be as embedded as a hot core (like IRS2E) and some fraction of the total number of the Lyman continuum photons emitted by the star will freely escape without ionizing the surrounding gas. If this is the case, the ionizing source would be of earlier spectral type.

\subsection{The Distance to IRS2}

Normally, one could derive the distance to W51d using its photometry in the NIR and the spectral type obtained above. However, we could not obtain a reliable photometry in the $H$ band from the NACO image. W51d seems to be saturated (as well as the object BBCDF4) and every attempt to reconstruct its PSF has failed. Moreover, this source seems to have strong excess emission and in this case we cannot derive its $H-K$ color nor assign its extinction. Without its $H-K$ color and extinction we cannot derive an estimate for its distance using spectroscopic parallax.

On the other hand, based on the spectral type presented in the previous section, we can estimate the distance to IRS2 using the radio data available in the literature. We can derive the number of photons emitted in the Lyman continuum by an O3 star and scale the distance until we reproduce the flux measured in the radio at a given wavelength. We need to keep in mind the limitations of this method, since it assumes that all photons emitted in the Lyman continuum are effectively ionizing the gas, and this may not be true as explained in the previous section. Some amount of the total number of photons may be escaping the UCHII while other photons are destroyed by dust.

Starting with equation (4) given by Kurtz et al. (1994) $(\xi=1$, i.e. all photons in the Lyman continuum effectively ionize the gas) we obtain the distance $d$ (in kpc) as a factor of the electron temperature $T_{e}$, the correction factor $\alpha\left(\nu, T_{e}\right)$, the frequency $\nu$ and the flux density $S_{\nu}$ as:

$$
d^{2}=1.32 \times 10^{-49} N_{C} \frac{\alpha\left(\nu, T_{e}\right)}{S_{\nu}(J y)}\left(\frac{\nu}{G H z}\right)^{-0.1}\left(\frac{T_{e}}{K}\right)^{0.5} .
$$

The critical parameter in the above equation is the number of photons emitted in the Lyman continuum $N_{C}$. Values for that parameter can vary an order of magnitude depending 
on which author is quoted. We derived $N_{C}$ as follow: for spectral types ranging from an O3 to an $\mathrm{O} 4$ star at the zero age main sequence (ZAMS) we took their effective temperature from Table 1 of Martins et al. (2005): 44,616 K for an O3 and 43,419 K for an O4. Using the Geneva models of Schaller et al. (1992) we obtained the bolometric magnitude $M_{b o l}$ for each star in the ZAMS (-8.94, O3 and -8.7, O4). We derived the stellar radius from the effective temperature above and its corresponding luminosity: 5.476 and $9.2 R_{\odot}$ for an $\mathrm{O} 3$ and 4.638 and $8.7 R_{\odot}$ for an $\mathrm{O} 4$. The radius and the luminosity calculated for a star in the ZAMS are significantly smaller than those quoted for main sequence stars and may be more reliable for W51d, given its young age. Finally, we used the radius for each star to obtain the number of ionizing photons emitted per unit time from the equation (9) and the ionizing fluxes $Q_{0}$ for each respective effective temperature also quoted in the Table 1 of Martins et al. (2005). According to these procedures an O3 can produce $1.86 \times 10^{49} \mathrm{~s}^{-1}$ and an O4, $1.44 \times 10^{49} \mathrm{~s}^{-1}$ in the ZAMS.

Returning to our equation (1), $S_{\nu}$ and $T_{e}$ quoted for W51d are: $5.35 \mathrm{Jy}$ and $10^{4} \mathrm{~K}$ at $\nu=15 \mathrm{GHz}$ (Wood \& Churchwell 1989) and $\alpha\left(\nu, T_{e}\right)=0.9767$ Mezger \& Henderson (1967). The radio flux of W51d is blended with that of $\mathrm{W}_{5} 1 \mathrm{~d}_{1}$ in data presented by Mehringer (1994), so it is overestimated and therefore cannot be used. The results are: for a ZAMS O3 star $d=5.8 \mathrm{kpc}$ and for an ZAMS O4 $d=5.1 \mathrm{kpc}$. We can compare our results with those obtained using the number of ionizing photons given by Martins et al. (2005) for main sequence stars, in this case the distance is $8.8 \mathrm{kpc}$ for an $\mathrm{O} 3 \mathrm{~V}$ and $7.4 \mathrm{kpc}$ for an $\mathrm{O} 4 \mathrm{~V}$.

We cannot take these results at their face value, given the limitations of the method described above. However, the results can be used as upper limits to the distance to IRS2. The distance to W51 North, which may be associated with IRS2, quoted in the literature were obtained through observations of proper motions of water masers and amounts to 8.3 $( \pm 2.5) \mathrm{kpc}$ (Schneps et al. 1981) and $6.1( \pm 1.3) \mathrm{kpc}$ (Imai et al. 2002). The upper limits obtained above are compatible (within the errors) with those obtained from the water masers if we consider the ionizing star of W51d an O3-O4 star in the ZAMS. However, these upper limits would make IRS2 part of W51 Main just as an effect of projection on the sky, given the distance of $2 \mathrm{kpc}$ obtained with spectroscopic parallax by Figuerêdo et al. (2008).

\section{Conclusions}

We presented $K$-band spectra of the embedded sources IRS2E and IRS2W/W51d in the compact HII region IRS2. The high angular resolution achieved by NIFS and Altair made possible the separation of the strong extended emission from the emission of the MYSO itself. It has proved a valuable tool in the study of MYSOs as we summarize our results 
presented in the previous sections as follow:

1) We identified the ionizing source of the UCHII region W51d as a massive star earlier than about type $\mathrm{O} 4$ based on the NIII multiplet (in emission), the HeII line (in absorption) and the absence of CIV in emission;

2) We detected compact (unresolved) $\mathrm{CO}$ overtone emission toward source IRS2E for the first time, suggesting the presence on an accretion disc around this source. The absence of radio emission and the presence of an accretion disk indicate that IRS2E is a massive YSO.

3) Combining the spectroscopy of W51d and its radio data, we found an upper limit to the distance to IRS2 between 5.1-5.8 kpc for an ionizing star of spectral type between O3-O4 in the ZAMS.

The fact that we detected two very massive stars, close in space, one which has cleared its surrounding material enough to probe its photosphere and another in an younger phase, deeply embedded in its dust cocoon and still surrounded by a disk, indicates that the evolutive timescale of MYSOs is extremely short.

CLB and AD are grateful to FAPESP (06/02467-0) and CNPq for financial support. PSC appreciates the continuing support from NSF. The authors wish to acknowledge Dr. Churchwell for making available the electronic radio map of W51 IRS2 and the anonymous referee for many valuable comments and suggestions.

Based on observations obtained at the Gemini Observatory, which is operated by the Association of Universities for Research in Astronomy, Inc., under a cooperative agreement with the NSF on behalf of the Gemini partnership: the National Science Foundation (United States), the Science and Technology Facilities Council (United Kingdom), the National Research Council (Canada), CONICYT (Chile), the Australian Research Council (Australia), CNPq (Brazil) and SECYT (Argentina). Partially based on observations made with ESO Telescopes at Paranal Observatories under program ID 71.C-0344

Facilities: Gemini:Gillet, NIFS, VLT, NACO.

\section{REFERENCES}

Barbosa, C.L., Damineli, A., Blum, R.D., \& Conti, P.S. 2003, AJ, 126, 2411 
Barbosa et al. 2008 in preparation

Bik, A. \& Thi, W.F. 2004, A\&A, 427, L13

Bik, A., Kaper, L., Hanson, M.M. \& Smits, M. 2005, A\&A, 440, 121

Bik, A., Kaper, L., \& Waters, L. B. F. M. 2006, A\&A, 455, 561

Blum, R. D., Barbosa, C. L., Damineli, A., Conti, P. S., \& Ridgway, S. 2004, ApJ, 617, 1167

Blum, R.D. \& McGregor, P. 2008, ApJ, accepted, arXiv:0802.2895v1 [astro-ph]

Churchwell, E. 2002, ARA\&A, 40, 27

Figuerêdo, E., Blum, R.D., Damineli, A., Conti, P.S., \& Barbosa, C.L. 2008, AJ, accepted, arXiv:0802.3558v1 [astro-ph]

Goldader, J. D. \& Wynn-Williams, C. G. 1994, ApJ, 433, 164

Hanson, M. M, Conti, P. S., \& Rieke, M. J. 1997, ApJ, 489, 698

Hanson, M. M., Luhman, K.L. \& Rieke, G.H. 2002, ApJS, 138, 35

Hanson, M. M., Kudritzki, R.-P., Kenworthy, M. A., Puls, J., Tokunaga, A. T. 2005, ApJS, 161,154

Imai, H., Watanabe, T., Omodaka, T., Nishio, M., Kameya, O., Miyaji, T., \& Nakajima, J. 2002, PASJ, 54, 741

Kraemer, K. E., Jackson, J. M., Deutsch, L. K., Kassis, M., Hora, J.L., Fazio, G.G., Hoffmann, W. F., \& Dayal, A. 2001, ApJ, 561, 282

Kumar, M. S. N., Kamath, U. S., \& Davis, C. J. 2004, MNRAS, 353, 1025

Kurtz, S., Churchwell, E., \& Wood, D.O.S. 1994, ApJS, 91, 659

Martin, A. H. M. 1972, MNRAS, 157, 31

Martins, F., Schaerer, D., \& Hillier, D.J. 2005, A\&A, 436, 1049

Mehringer, D. M. 1994, ApJS, 91, 713

Mezger, P.G. \& Henderson, A.P. 1967, ApJ, 147, 471

Najita, J., Carr, J. S., Glassgold, A. E., Shu, F. H. \& Tokunaga, A. T. 1996, ApJ, 462, 919 
Okamoto, Y. K., Kataza, H., Yamashita, T., Miyata, T., \& Onaka, T., ApJ, 553, 254

Okumura, S., Mori, A., Watanabe, E., Nishihara, E., \& Yamashita, T. 2001, AJ, 121, 2089

Schaller, G., Schaerer, D., Meynet, G., \& Maeder, A. 1992, ApJS, 96, 269

Schneps, A., Lane, A. P., Downes, D., Moran, J. M., Genzel, R., \& Reid, M.J. 1981, ApJ, 249,124

Watson, A.M. \& Hanson, M.M. 1997, ApJ, 490, L165

Wilson, T. L., Mezger, P. G., Gardner, F. F., \& Milne, D. K. 1970, ApJ, 5, 99

Wood, D.O.S. \& Churchwell, E.1989, ApJS, 69, 831

Zinnecker, H. \& Yorke, H. W. 2007, ARA\&A, 45, 481 


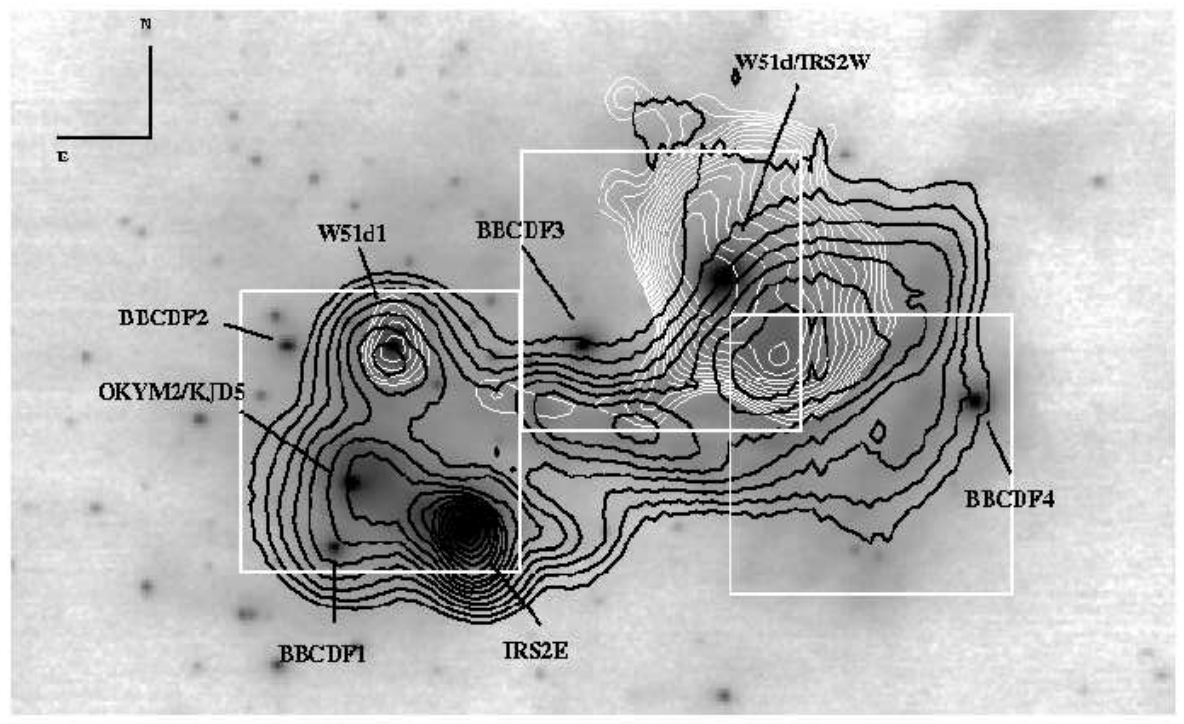

Fig. 1.- Finding chart of IRS2. The white contours are the radio continuum emission at 2 $\mathrm{cm}$. The black contours represent the MIR emission at $12 \mu \mathrm{m}$. White squares represent the field of view of NIFS $\left(3^{\prime \prime} \times 3^{\prime \prime}\right)$ and indicate the positions of the three pointings of our program. The sources labeled are those which we have "pseudo long-slit" spectra. All contours are in arbitrary units. 


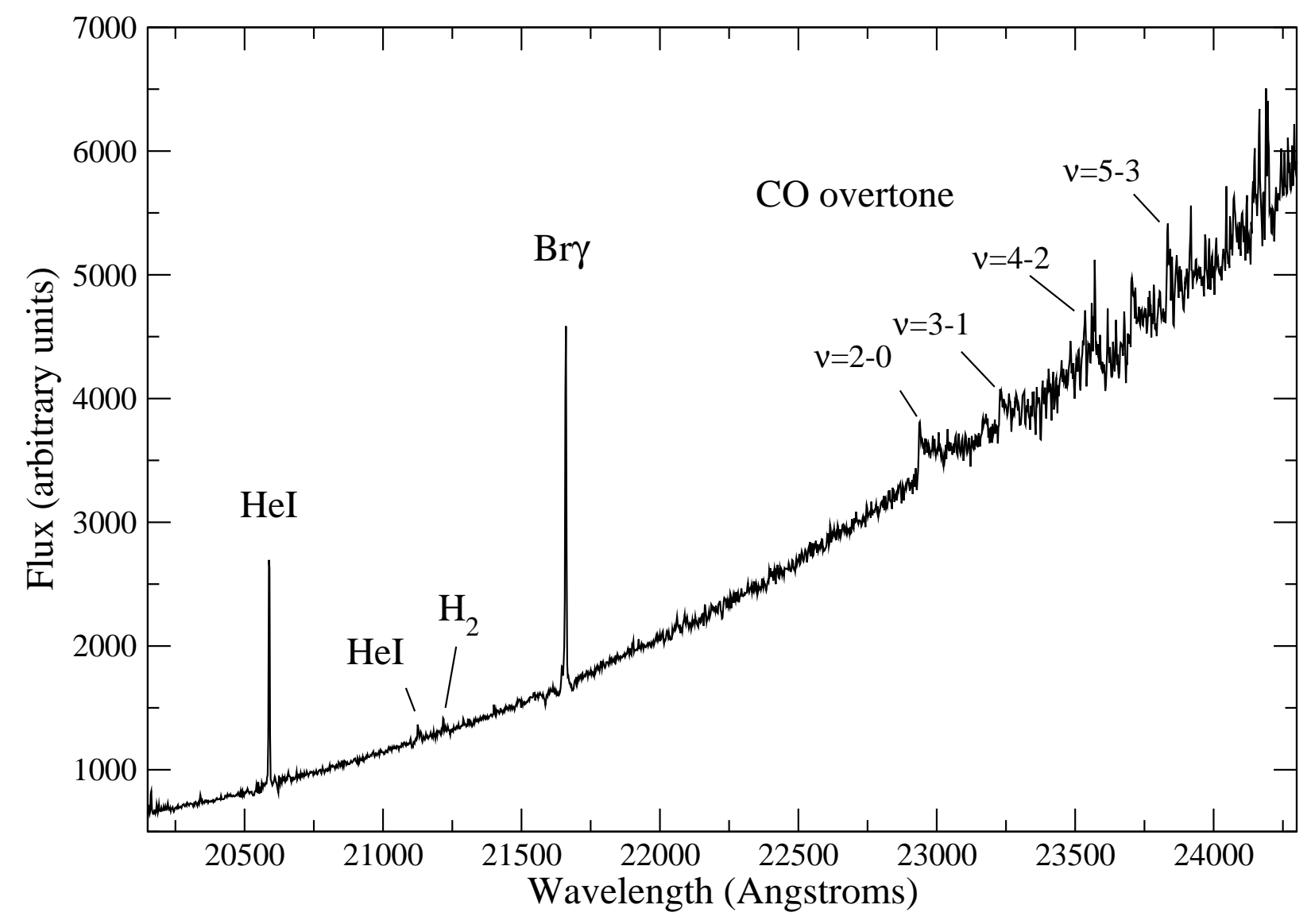

Fig. 2.- $K$-band spectrum of source IRS2E. After the spectrum was extracted it was multiplied by a black body of $9000 \mathrm{~K}$ to recover the true spectral shape of the source. The flux is in arbitrary units. 


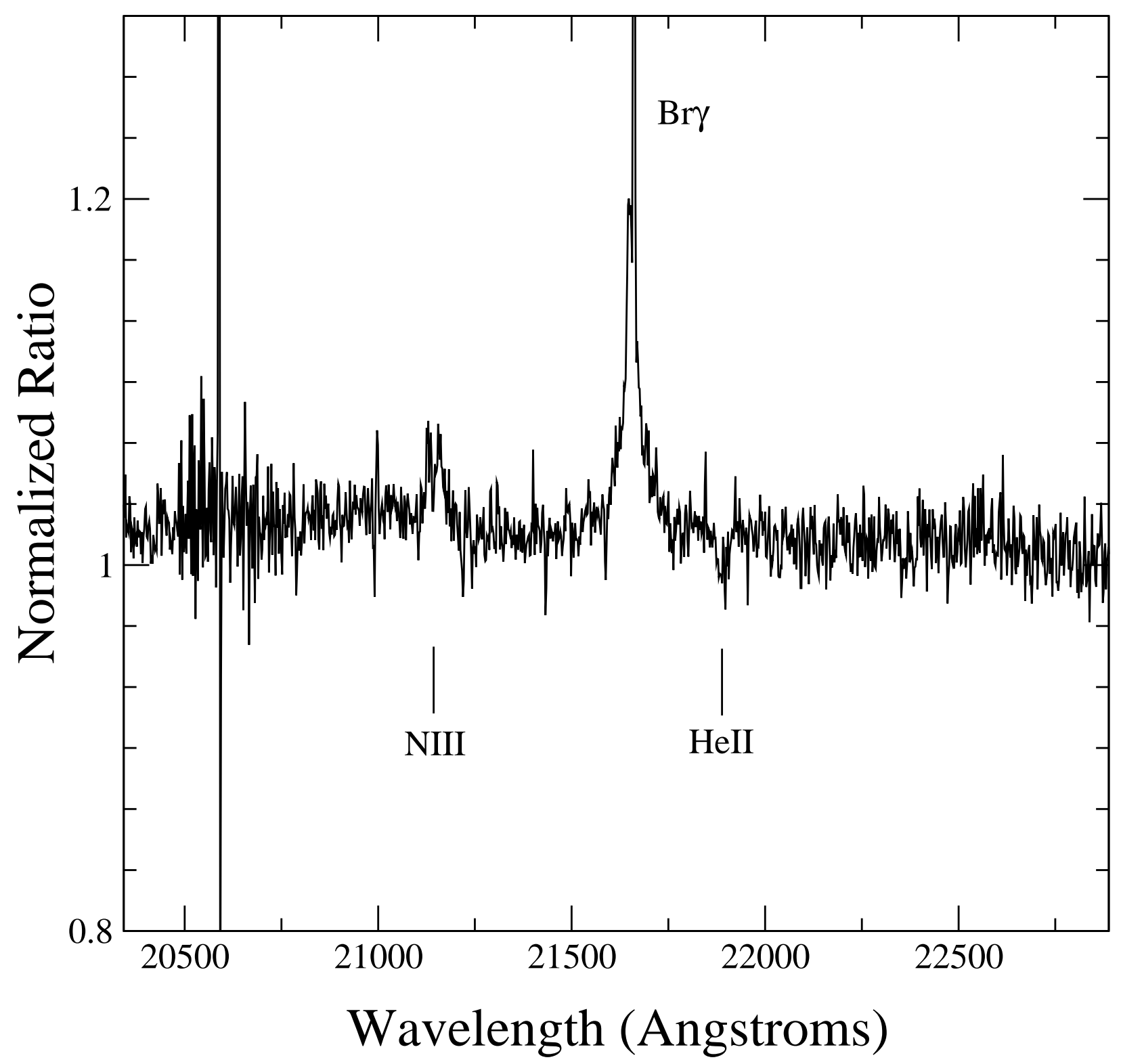

Fig. 3.- $K$-band spectrum of source W51d. This spectrum was zoomed to show the NIII multiplet and HeII. The thin line near $20587 \AA$ is a residual feature produced after the correction of the telluric lines. 